\title{
Los impactos de la descentralización de la enseñanza y la investigación en el Cinvestav de 1961-2015: estudio bibliométrico
}

\author{
María Elena Luna-Morales* \\ Evelia Luna-Morales* \\ Miguel Ángel Pérez-Angón**
}

Artículo recibido:

5 de abril de 2018

Artículo aceptado:

11 de marzo de 2019

Artículo de investigación

\section{Resumen}

Se usa la información registrada en Web of Science (WoS) de 1961-2015 para determinar los crecimientos de producción e impacto científico de las unidades, departamentos y laboratorios que se crearon como parte del proceso de descentralización del Centro de Investigación y Estudios Avanzados (Cinvestav). El estudio se complementa con variables como número de investigadores, departamentos, graduados, posgrados de calidad, distinciones y reconocimientos. Para medir los incrementos de estas variables se aplicaron, además de WoS, el Atlas de la Ciencia en el Cinvestav, el catálogo colectivo de bibliotecas de la institución, los anuarios, y los sitios web de la administración, de las

* Coordinación General de Servicios Bibliográficos, Cinvestav-IPN, México meluna@cinvestav.mx eluna@cinvestav.mx

** Departamento de Física, Cinvestav-IPN, México_mperez@fis.cinvestav.mx

INVESTIGACIÓN BIBLIOTECOLÓGICA, vol. 33, núm. 81, octubre/diciembre, 2019, México, ISSN: 2448-8321 pp. 57-87 
unidades y departamentos del Cinvestav. Las aportaciones se miden en porcentajes comparando la Unidad Zacatenco con el resto de las unidades de este centro. De esta manera se puede observar que el Cinvestav se fundó en 1961 con la Unidad Zacatenco en la Ciudad de México, a partir de 1980 inició su proceso de descentralización con la apertura de ocho unidades foráneas y una sede. El crecimiento de la planta de investigadores en las unidades foráneas ha sido tal que actualmente iguala en producción científica y formación de recursos humanos de maestría y doctorado a la planta académica de la Unidad Zacatenco.

Palabras clave: Cinvestav; DescentralizaciónCiencia; Estudio-Bibliométrico

The Impacts of descentralization of teaching and research in the Cinvestav of 1961-2015: Bibliometric study María Elena Luna-Morales, Evelia Luna-Morales and Miguel Ángel Pérez-Angón

\section{Abstract}

The information registered in Web of Science (WoS) from 1961-2015 is used to determine the production growth and scientific impact of the units, departments and laboratories that were created as part of the decentralization process of the Center for Research and Advanced Studies (Cinvestav). The study is complemented by variables such as: number of researchers, departments, graduates, certified graduate programs, distinctions and recognitions. In order to measure the increments of the variables, besides WoS, were also applied the Atlas of Science in the Cinvestav, the collective library catalog of the institution, the yearbooks, the websites of the administration and the units and departments of the Cinvestav. Contributions are measured in percentages by comparing the Zacatenco Unit versus the rest of other units. This way, it can be observed that the Cinvestav was founded in 1961 with the Zacatenco Unit in Mexico City and, starting in 1980, it began its decentralization process with the opening of eight foreign units and a second metropolitan office. The growth of the faculty plant in these units has been such that it currently matches the scientific production and training of human resources of masters and doctorates in the Zacatenco unit.

Keywords: Cinvestav; Descentralization-Science; Bibliometric-Study. 


\section{INTRODUCCIÓN}

-1 Centro de Investigación y de Estudios Avanzados del IPN (CinvesEtav-IPN) se concibe durante el periodo considerado como el umbral de la revolución científico-técnica (Huerga-Melcón, 2012), un evento que sustenta el progreso económico en las ventajas que ofrece el desarrollo científico y tecnológico alcanzado durante el siglo XX. En este contexto, el 17 de abril de 1961 se crea el Cinvestav (García-Ramos, 1970) para impulsar la ciencia en el país dando continuidad al desarrollo generado desde finales de la década de los años 50, periodo en el que se establecieron diversas instituciones en el país orientadas al avance de la ciencia.

El Cinvestav nace de las ideas de distintas personalidades que consideraron que al país le hacía falta consolidar el desarrollo de la ciencia a través de una institución dedicada a cumplir con distintos objetivos: "preparar investigadores, profesores especializados y expertos en diversas disciplinas científicas y técnicas, así como en la solución de problemas tecnológicos del país" (Ibarrola, 2002: 11). El Cinvestav se creó bajo la percepción de su último impulsor y primer director, Arturo Rosenblueth, dando lugar a uno de los primeros Centros de investigación establecidos en el país y en América Latina (Martínez-Palomo, 2011).

El Cinvestav inició actividades con un grupo muy reducido de profesores, quienes realizaban investigación en tres campos de estudio: fisiología y biofísica, matemáticas, y física. En 1962 se integraron dos áreas más: bioquímica e ingeniería eléctrica, dando lugar a la primera estructura departamental con la que contó la institución. Con los años la estructura se fue modificando hasta quedar dividida en 32 departamentos distribuidos en las cuatro áreas que actualmente trabaja este centro: Ciencias Biológicas y de la Salud (CBS), Ciencias Exactas y Naturales (CEN), Ciencias Sociales y Humanidades (CSH), Tecnología y Ciencias de la Ingeniería (TCI). Esta estructura se reforzó con la descentralización de la ciencia en México, evento que comenzó durante la década de los años 70 y se prolongó hasta el 2000 (Larqué-Saavedra, 2016), lo anterior como parte de las políticas científicas implementadas por el Consejo Nacional de Ciencia y Tecnología (Conacyt), una medida que permitiría, por un lado, llevar la ciencia hacia otros estados y, por otro, cooperar con la industria instalada en la región (Ureña, 2016).

El Cinvestav, al igual que otras instituciones, ha contribuido con la instalación de nuevas sedes en distintos puntos del país. Durante los años 70 se crearon en espacios externos a Zacatenco los departamentos de Investigaciones Educativas y Matemática Educativa. En los 80 se establecieron cinco unidades foráneas: Mérida, Irapuato, Saltillo, Laboratorio Tlaxcala y 
Guadalajara (Luna-Morales, 2013); en los periodos 1990 y 2000 se fundaron otras tres: Querétaro, Monterrey, Tamaulipas y se reinstala la Sede Sur en la Ciudad de México. De esta manera se abren nuevas líneas de investigación, se amplían y fortalecen las ya existentes colaborando con la industria instalada en las entidades federativas donde se establecieron, además del beneficio que ofrecerían a la sociedad. De esta manera, la unidad Mérida aborda temas de investigación como recursos del mar, física aplicada y ecología humana; Irapuato realiza investigación en biotecnología y bioquímica, ingeniería genética y genómica para la biodiversidad; Saltillo se especializa en ingenierías: cerámica, metalúrgica, robótica y manufactura, y recursos naturales y energía; Tlaxcala es responsable de la biología de la reproducción animal; Guadalajara cubre ingenierías, computación, comunicaciones, diseño y sistemas eléctricos; Querétaro se responsabiliza de la investigación en ciencias de los materiales; Monterrey trabaja con bioquímica y biofísica de fluidos y membranas, biología molecular y celular, probabilidad y estadística biomédicas, así como educación en ciencias; Tamaulipas se relaciona con inteligencia computacional y tecnología para la gestión de datos, la Sede Sur integra una parte de lo que es matemática educativa y farmacobiología y, por último, Zacatenco integra los campos de investigación más tradicionales: fisiología, biofísica, neurociencias, biología celular, patogénesis molecular, genética y biomedicina molecular, farmacología y toxicología, física, química, ingenierías, biotecnología, matemáticas y matemática educativa. Aunque el Cinvestav se funda en 1961, es hasta la década de los años 70 cuando se definen sus principales líneas de investigación (Campos-Ríos y Martínez de Ita, 2011).

\section{Descentralización en México}

El tema de la descentralización en México y en América Latina no es algo nuevo: desde la década de los años 80 surgieron los primeros proyectos en torno a la desvinculación política y de gobierno. No obstante, es a mitad de los años 90 cuando se logra mayor impacto como consecuencia del desarrollo de diversos eventos ocurridos particularmente en el ámbito gubernamental (cambios de gobierno y diversidad de partidos políticos). Actualmente, los gobiernos estatales ya pueden participar en temas de educación, salud, vivienda e infraestructura social (Cabrero-Mendoza, 2000). Situación similar presentan los municipios, que también tienen representación para conseguir ventajas para las comunidades que representan. A pesar de los cambios y logros alcanzados, algunos aspectos se mantienen igual, como es el caso del sistema fiscal que continúa centralizado, impidiendo que los recursos se muevan con la fluidez y llegan a los estados que lo requieren. 
Jaramillo-Cardona (2010) define la descentralización como un instrumento generador de desarrollo regional dado que se comparten responsabilidades entre los distintos niveles de gobierno a fin de resolver problemas específicos que afectan a la sociedad. La descentralización forma parte del conjunto de políticas públicas establecidas por el Estado orientadas a mejorar la salud, educación, desarrollo científico y tecnológico, entre otros aspectos. Este es el medio que gobierno y sociedad tienen para abrir espacios y mejorar las condiciones de vida de la población, así como generar ventajas económicas en una región específica. Con la descentralización se busca otorgar autonomía, responsabilidad y recursos a niveles gubernamentales de menor jerarquía.

Illán-Sailer (2006) clasifica la descentralización en tres tipos, según los objetivos a seguir: 1) políticos, espaciales (territoriales), 2) administrativos, y 3) económicos. De acuerdo con Fernández-Ruíz (2010), la descentralización territorial se caracteriza por asignar aspectos del ejercicio de funciones del poder público a territorios específicos, dándoles personalidad jurídica propia. En nuestro país son entes territoriales políticamente descentralizados, los estados de la República y los municipios. La descentralización administrativa otorga a los entes (sujetos) relativa autonomía orgánica que les permita realizar sus actividades, y se implementa mediante la creación de personas jurídicas.

La descentralización fiscal pretende dinamizar las relaciones fiscales intergubernamentales, fortaleciendo el sistema hacendario, buscando con ello un equilibrio mediante la transferencia de recursos federales hacia las entidades y los municipios por medio de un compromiso entre los gobiernos federal y estatal.

Esta nueva forma de convivencia entre el Estado y la sociedad permite evaluar las contribuciones y logros a través de diversos medios: fiscales, transparencia de acciones por parte del gobierno, participación ciudadana, asignación de recursos y el rendimiento de cuentas (Azfar et al., 1999). Sin embargo, determinar los logros no es fácil; en el caso de la descentralización institucional ligada al progreso de la ciencia se recurre generalmente a los métodos bibliométricos, informétricos y cienciométricos, según los fines del estudio. Un análisis similar de tipo bibliométrico registró resultados positivos en el periodo de descentralización de la ciencia mexicana en el caso de una política pública (Programa de Mejoramiento del Profesorado, PROMEP), aplicada a la contratación de nuevos investigadores en universidades públicas estatales (López-Olmedo et al., 2017). 


\section{Institucionalización y descentralización de la ciencia en México}

Los esfuerzos por institucionalizar y profesionalizar la ciencia en México tienen una trayectoria que parte de la época colonial. La etapa porfirista marca momentos fundamentales por la incorporación de técnicos y científicos a las estrategias de cambio que se buscaban. Es por ello que en este periodo se logró visibilidad para la ciencia y se crearon diversas sociedades científicas en el país, entre ellas la Sociedad Antonio Alzate, donde se profesionalizó por primera vez la ciencia, pues se pagó por desarrollar el trabajo de investigación (Saldaña-González y Azuela-Bernal, 1994). Los distintos eventos históricos acontecidos a nivel nacional señalan que ha sido a través del Estado que se ha logrado institucionalizar y profesionalizar la ciencia en el país (Saldaña-González, 1990), por eso en México la ciencia es un producto esencialmente del Estado.

Por otro lado, en México la ciencia está ligada a las universidades públicas por el empuje que la educación tuvo durante la estancia de José Vasconcelos al frente de la Secretaría de Educación Pública (SEP). Es por ello que el proceso de institucionalización y profesionalización de la ciencia surge desde las universidades, sobre todo, la Universidad Nacional Autónoma de México (UNAM); a partir de su autonomía, logró fundar los primeros institutos y centros de investigación, así como el desarrollo e implementación de los primeros programas de enseñanza en física y matemáticas (Gutiérrez-Serrano, 1997).

Durante los años 60 se continúa con la creación de diversas instituciones y centros de investigación en el país. Finalmente, se concreta la institucionalización y profesionalización de la ciencia con la creación de la Coordinación de la Investigación Científica (CIC), que posteriormente cambió de nombre por Instituto Nacional de Investigación Científica (INIC) (Tinajero-Villavicencio, 2005).

En 1969, por mandato de la Secretaría de la Presidencia el INIC realizó un diagnóstico sobre la situación que presentaba la ciencia y la tecnología en México. En consecuencia, el Estado determinó la necesidad de instalar un organismo que sistematizara la actividad científica nacional, y en 1970 se funda el Conacyt, instancia responsable de poner las bases en materia de ciencia y tecnología en el país.

Según Retana-Guiascón (2009), entre las primeras acciones que se propuso el Conacyt fue establecer 15 centros e institutos de investigación en diferentes entidades del país. El fin era resolver los problemas que existían en el ámbito nacional relacionados con "aprovechamiento de recursos, salud, alimentación, producción agropecuaria y forestal, educación, industrialización, comercio exterior y desarrollo rural" (50). Para incentivar, el gobierno federal puso en acción algunas iniciativas: la Ley para Coordinar y Promover 
el Desarrollo de la Ciencia y la Tecnología, el Programa Nacional para el Desarrollo de la Educación Superior, y la Descentralización y Regionalización de Programas de Posgrado e Investigación Científica y Tecnológica.

Para dar continuidad a las acciones antes señaladas, durante el periodo de los años 90 se inició un proceso de reestructuración institucional por medio de la implementación de diversas acciones, entre las que destacan la fundación del Sistema SEP-Conacyt, constituido por 29 centros de investigación que cubren las áreas científica, tecnológica y social, instalados en distintos estados del país; la integración de los Sistemas Regionales de Investigación con la idea de apoyar las necesidades locales mediante proyectos de investigación como el PROMEP, para incrementar la formación de recursos humanos y producción científica de las universidades estatales por medio del otorgamiento de tiempos completos para los profesores, así como la constitución de los Consejos Estatales de Ciencia y Tecnología en la mayor parte de los estados (Campos-Ríos y Martínez de Ita, 2011). El proceso de descentralización en México sigue aplicando acciones orientadas a hacer más equitativas las actividades, sobre todo que la sociedad logre beneficios concretos.

\section{La bibliometría en la evaluación de la actividad cientifica}

El papel de la ciencia y tecnología en el crecimiento económico es fundamental. Los descubrimientos científicos y su aplicación en la generación de tecnologías impactan sobre todo en el desarrollo económico y social del país que la produzca y mantenga actualizada (Romo-Murillo, 2006). Las naciones que logran adecuados progresos científicos y tecnológicos son las que tienen las mejores economías a nivel mundial (Peña-Ocando, 2011). En este sentido son distintas las razones por las que se evalúa la ciencia, entre otras, determinar fortalezas y debilidades, determinar la eficiencia y la eficacia del sistema que organiza la actividad científica de un país, definir los alcances de la producción y el impacto en citas en distintos ámbitos (Vanti, 2010). Además, es el medio por el que se obtiene apoyo en la investigación, promociones, distinciones, premios y reconocimientos, estos últimos señalados por Merton (1988) como parte del sistema de recompensas y evaluación de la ciencia. En general son diversos los aspectos que se toman en cuenta para evaluar, sobre todo porque repercuten en la toma de decisiones y en la aplicación y modificación de políticas científicas nacionales e institucionales que, por lo general, conducen a mejorar las formas de organizar la actividad científica y tecnológica (Velasco et al., 2012).

La bibliometría forma parte de los métodos que existen para evaluar la actividad científica. Este método tiene sus orígenes en los años 20 del siglo 
pasado, etapa en la que se dan a conocer los primeros conceptos y definiciones y se desarrollan los primeros trabajos orientados al estudio de la producción científica en campos específicos del conocimiento. Pritchard (1969: 349) fue el primero en dar a conocer el término bibliometría, definiéndolo como "la aplicación de los métodos estadísticos y matemáticos que permiten definir los procesos de la comunicación escrita, la naturaleza y el desarrollo de las disciplinas científicas mediante técnicas de recuento y análisis de dicha comunicación”. A partir de este suceso se han dado a conocer más estudios respaldados en el método bibliométrico a través de las teorías cuantitativas y cualitativas, donde la cuantitativa permite examinar los datos de manera numérica en complemento con el apoyo estadístico (Mendoza y Garza, 2009).

El Cinvestav tiene más de 55 años realizando actividades de investigación y formación de investigadores a través de las unidades y laboratorios instalados en las distintas entidades del país. En este sentido, se consideró conveniente llevar a cabo un estudio para determinar los efectos que ha generado la descentralización en la enseñanza y la investigación científica que se realiza en el Cinvestav, así como el crecimiento de la producción e impacto científico registrado en las bases de datos Web of Science (1961-2015). La hipótesis sugiere que Zacatenco es la unidad más productiva debido a que tiene más tiempo e integra un número mayor de investigadores, departamentos y programas de posgrado.

El trabajo se apoya en el método bibliométrico cuantitativo que permite examinar los datos de manera numérica, basado en la medición de indicadores de producción e impacto (Mendoza y Garza, 2009), en complemento con otros indicadores como número de investigadores, departamentos, graduados, posgrados y distinciones. Existen otras teorías que pueden ayudar a determinar las estructuras de los grupos de investigación, la colaboración científica, los temas sobresalientes y la identificación de los principales productores de la ciencia. No obstante, en este primer análisis pretendemos dar a conocer únicamente tendencias cuantitativas para ir dejando pruebas de los progresos de las instituciones de investigación en el país, considerando que hay pocos estudios orientados a presentar este tipo de avances (Hernández-García y Navarro-Contreras, 2017).

Las unidades externas del Cinvestav están haciendo aportaciones significativas que casi empatan con Zacatenco a pesar de que hay unidades que todavía no logran consolidar sus estructuras de organización, lo que les permitiría mejorar su contribución. La descentralización en el Cinvestav ha dado buenos resultados a pesar de las limitaciones del Estado con respecto a recursos orientados a la investigación. 


\section{Metodología}

A partir de búsquedas hechas en las bases de datos Web of Science (WoS) durante marzo de 2016, se recopilaron los trabajos publicados con dirección al Cinvestav de 1961-2015. La búsqueda se llevó a cabo por dirección considerando las más de 25 formas en que aparece registrado en los documentos publicados. Con esta estrategia se obtuvieron las publicaciones de otras instituciones que también llevan el nombre de Cinvestav o Centro de Investigación y Estudios Avanzados, y por ello fue necesario revisar uno a uno los trabajos para identificar los que correspondían al Cinvestav-IPN. Las citas consideradas en este análisis también abarcan el tiempo de búsqueda ya señalado; sin embargo, los trabajos publicados en los últimos años del estudio, los dados a conocer entre 2013-2015, todavía no recuperan todas las citas que les corresponden debido a que dependen del índice de inmediatez de las revistas donde se difundieron los documentos (Hernández-Arias, 2013). Algunas revistas registran citas inmediatamente e incluso el mismo año de publicación del trabajo, otras tardan más tiempo, por ello conviene aclarar que esta situación generalmente provoca una caída de citas en los últimos años analizados.

Otro paso importante fue la desagregación del campo de dirección, que se llevó a cabo hasta que se completaron los datos de adscripción de los autores (unidad, departamento, sección y laboratorio, entidad federativa y país) debido a que gran parte de los trabajos no los incluyen. En este caso fue necesaria la consulta en fuentes de información como Anuarios-Cinvestav, documentos sobre la historia del Cinvestav, páginas web de los departamentos, texto completo de los artículos publicados y bases de datos: Scopus, SciFinder y Google Scholar. Cabe aclarar que las colaboraciones se contabilizaron para cada una de las unidades y departamentos participantes, tanto en trabajos como en citas.

Del Atlas de la Ciencia en el Cinvestav (ACC) 1961-2010 (http://transdisciplinario.cinvestav.mx/Portals/transdisciplinario/SiteDoc/Seminar\%20Memory/Presentaciones/Atlas-Cinvestav_ElenaLuna.pdf se tomaron los nombres de los investigadores por departamentos, secciones y laboratorios. Para completar los últimos cinco años (2011-2015) se revisaron las listas de profesores afiliados al Cinvestav en la página web http://www.cinvestav.mx/Investigacion/ListadeInvestigadores.aspx. La consulta y revisión de estas fuentes dio como resultado una lista definitiva de investigadores que integran la planta académica de la institución. La relación se ordenó en forma alfabética, lo que facilitó la clasificación de profesores por género (femenino y masculino).

Para conocer el número de estudiantes graduados se revisó el catálogo colectivo del Cinvestav mediante búsquedas por año, departamento, limitando 
al formato de tesis. La lista de distinciones y reconocimientos recibidos por investigadores y estudiantes se obtuvo a través de varias búsquedas: a) Anuarios-Cinvestav, b) currículos de investigadores, c) resultados de ganadores por los nombres de los premios, buscando año por año, por ejemplo: Príncipe de Asturias, L’Oreal Unesco, Premio TWAS, AgroBio, Presea Lázaro Cárdenas, Canifarma México, entre otros reconocimientos otorgados a nivel nacional, regional e internacional. Los Programas Nacionales de Posgrado de Calidad (PNPC) de maestría y doctorado que el Cinvestav tiene reconocidos se tomaron del sitio oficial del Conacyt (https://www.conacyt.gob.mx/index.php/becas-y-posgrados/programa-nacional-de-posgrados-de-calidad).

Por último, para facilitar la interpretación de la Figura 11 referente a trabajos con 100 o más citas, investigadores, departamentos, posgrados y reconocimientos, se ajustaron a escala logarítmica de base dos y base tres, respectivamente, debido a la dispersión de valores en las mismas variables. Con esta escala es posible apreciar de mejor manera las diferencias entre los valores más pequeños y los más grandes.

\section{Resultados}

\section{Aspectos generales}

El Cinvestav está conformado por siete unidades, una sede y dos laboratorios instalados en distintas entidades federativas; en conjunto reúne un total de 655 investigadores, $95 \%$ de ellos pertenecen al Sistema Nacional de Investigadores (SNI), distribuidos en 33 diferentes dependencias del Cinvestav. En términos globales los profesores han formado a 8182 maestros y 3440 doctores. El Cinvestav en 55 años de vida académica y de investigación ha recibido más de 1000 distinciones y reconocimientos, $24 \%$ de procedencia internacional y 76 \% nacional. Los trabajos registrados en WoS en el periodo 1961-2015 suman un total de 25995 con 340339 citas. Los documentos están distribuidos en 3369 distintos títulos de revistas, de los cuales $80 \%$ tienen factor de impacto (FI) hasta 2015, versión de revisión del FI. El 20 \% restante no forma parte del Journal Citation Reports (JCR), entre estos últimos aparecen títulos nacionales y regionales que en algún momento fueron fundamentales para la divulgación de la actividad científica y que por alguna razón quedaron fuera del JCR, ya sea porque la revista se dio de baja, cambió de nombre, se fusionó o fue absorbida por otra. 


\section{Producción e impacto por unidad}

La Figura 1 presenta la producción del Cinvestav por serie anual, se observa la aportación de la unidad Zacatenco desde los primeros años de creación. A partir de los años 80 comienza a distinguirse la contribución de las primeras unidades del Cinvestav: Mérida, Irapuato, Guadalajara, Saltillo, Querétaro y Sede Sur.

La mayor parte de las unidades del Cinvestav se crearon en el periodo de los años 80 y 90. Las más recientes son Monterrey y Tamaulipas, la primera con 11 años de actividad y la segunda con 10 a 2015; ambas contribuyen con producción desde sus primeros años de incorporación.

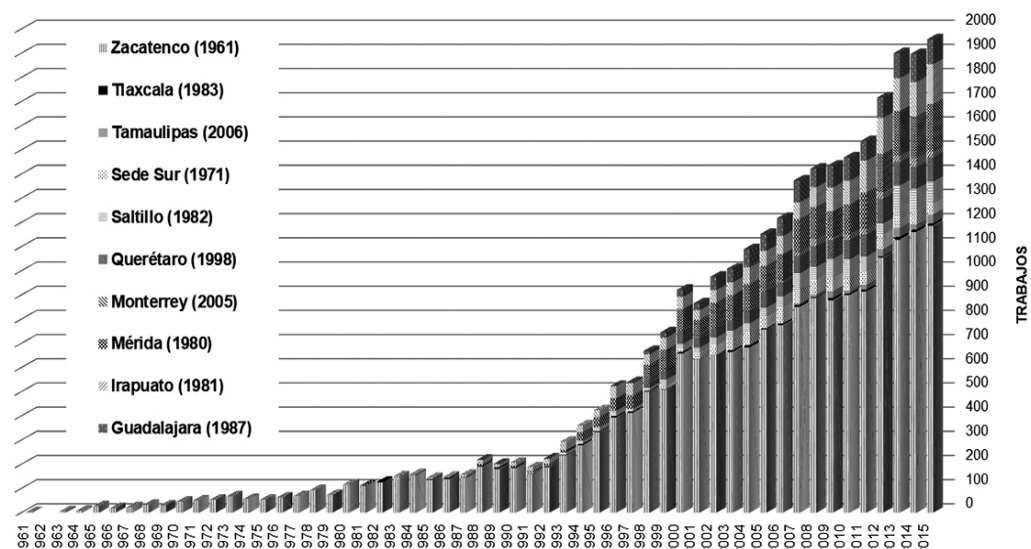

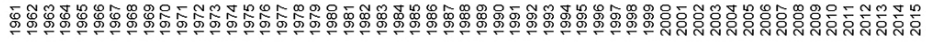

AÑos

HIgura 7. Uistribucion por serıe anual de Ios trabajos publicados por unidades del Linvestav: 1961-2U15

La Figura 2 muestra las citas por unidades y por serie anual, el grueso de las citas proviene de los trabajos publicados en Zacatenco. No obstante, contribuyen de manera extraordinaria Mérida e Irapuato, que desde sus primeros años de creación ya recuperan citas, mismas que se incrementan durante el periodo de los años 90. Las unidades Guadalajara, Saltillo, Querétaro y Sede Sur registran importantes contribuciones a finales de los 90. Las unidades Tamaulipas, Tlaxcala y Monterrey muestran menor número de citas debido a que se trata de las unidades más recientes, excepto Tlaxcala que a pesar de los años que tiene no ha logrado consolidar sus estructuras de organización científica. 


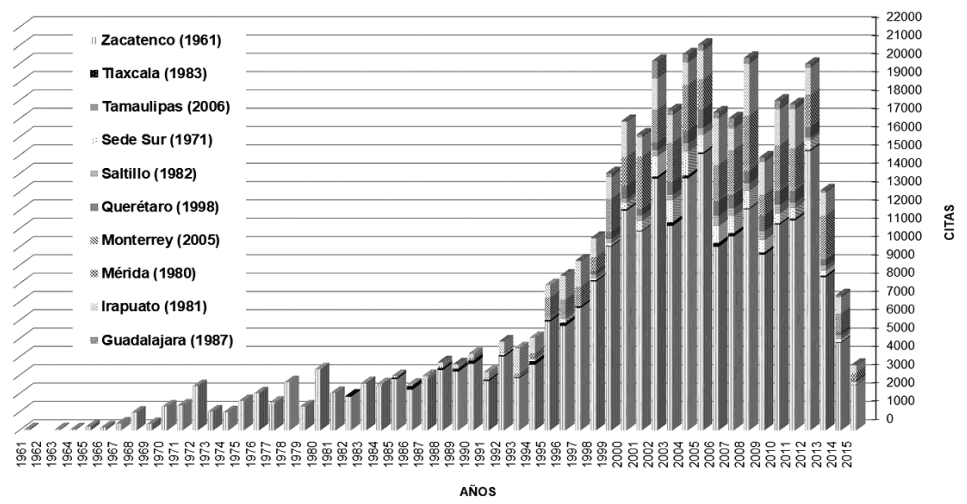

Figura 2. Citas por serie anual para cada una de las unidades Cinvestav: 1961-2015

\section{Trabajos y citas por quinquenio}

La Figura 3 presenta los porcentajes de trabajos por quinquenio entre Zacatenco y el resto de las unidades del Cinvestav. Como se observa, hasta 1975 Zacatenco se mantiene como la única unidad, por tanto su aportación es de $100 \%$, aun cuando en el quinquenio 1971-1975 se integra el área de matemática educativa en un espacio externo a Zacatenco y en el quinquenio 19761980 se incorpora unidad Mérida. En este último quinquenio se muestran cambios en las contribuciones: Zacatenco registra $99.5 \%$ de los trabajos publicados y las demás unidades hasta el momento instaladas $0.5 \%$. En la década de 1981-1990 se incorporan Irapuato, Saltillo, Tlaxcala y Guadalajara, lo que produce nuevos cambios en la producción sobre todo en el segundo quinquenio cuando Zacatenco aparece con $89.4 \%$ y el resto de las unidades $10.6 \%$. Querétaro se crea en 1998, Monterrey y Tamaulipas en la segunda mitad de la década de los años 2000, modificando otra vez las aportaciones, de manera que las unidades foráneas alcanzan casi $40 \%$ de la producción y Zacatenco poco más de $60 \%$.

La Figura 4 muestra el comportamiento de las citas por quinquenio, se puede ver que Zacatenco desde 1961 hasta 1975 es el único con citas. Cambia el esquema de 1976-1980 porque Zacatenco genera $99.1 \%$ y el resto de las unidades $0.9 \%$. En el segundo quinquenio de los años 80 , las unidades en provincia y Sede Sur acumulan $10.8 \%$ del total de las citas. Con la incorporación de nuevas unidades, las aportaciones de los grupos foráneos son mayores y esto implica que Zacatenco reduce su porcentaje. En el quinquenio 2006-2010 es cuando más se unen las líneas entre Zacatenco y el resto de las 
unidades. La separación del último periodo analizado tiene una explicación: no se ha recuperado el total de las citas para los trabajos publicados entre 2013-2015. Es muy probable que, de reunirse las citas faltantes, las líneas se vuelven a juntar, porque hay campos de investigación que se están consolidando como es el caso de física de partículas y campos, genética y biología molecular, al igual que diversas áreas de las ingenierías computacionales.

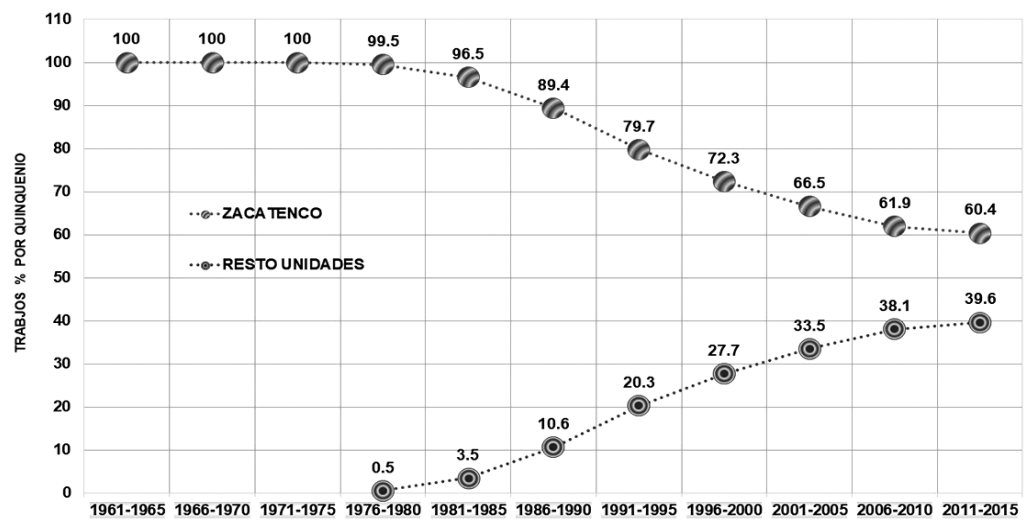

Figura 3. Trabajos por quinquenio Zacatenco vs. el resto de las unidades: 1961-2015

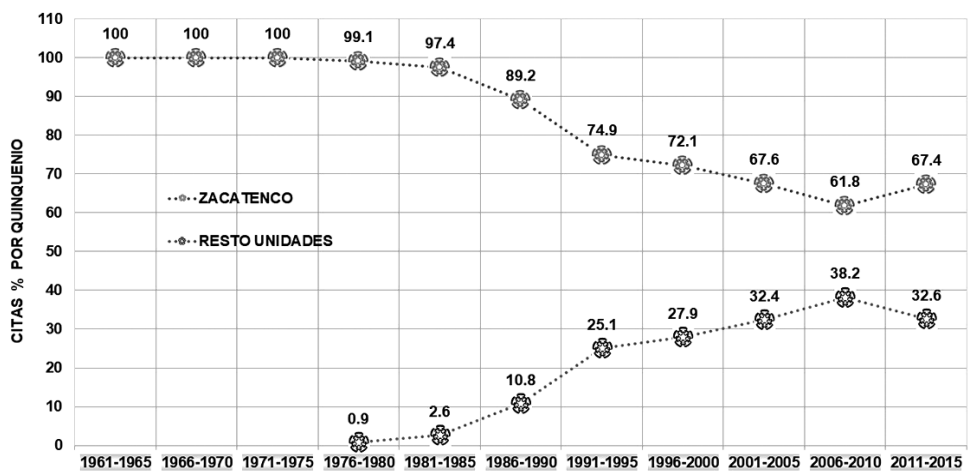

Figura 4. Citas por quinquenio Zacatenco vs. el resto de las unidades: 1961-2015 


\section{Profesores, investigadores}

De acuerdo con la Figura 5, Zacatenco es el único que registra profesores-investigadores durante los 10 primeros años. A principios de los años 70 aparecen los primeros profesores en unidades externas a Zacatenco, adscritos al Departamento y a la Sección de Matemática Educativa; ambas dependencias reúnen $4.8 \%$ del total de investigadores en el quinquenio 1971-1975. La Figura 5 muestra la forma en que va creciendo el porcentaje de investigadores en las unidades externas entre un quinquenio y otro, a tal grado que en los últimos dos lustros ocurre un acercamiento muy importante entre las variables analizadas. Cabe aclarar que en realidad se trata de una estabilización en el número de investigadores, misma que se está produciendo desde el quinquenio 1996-2000.

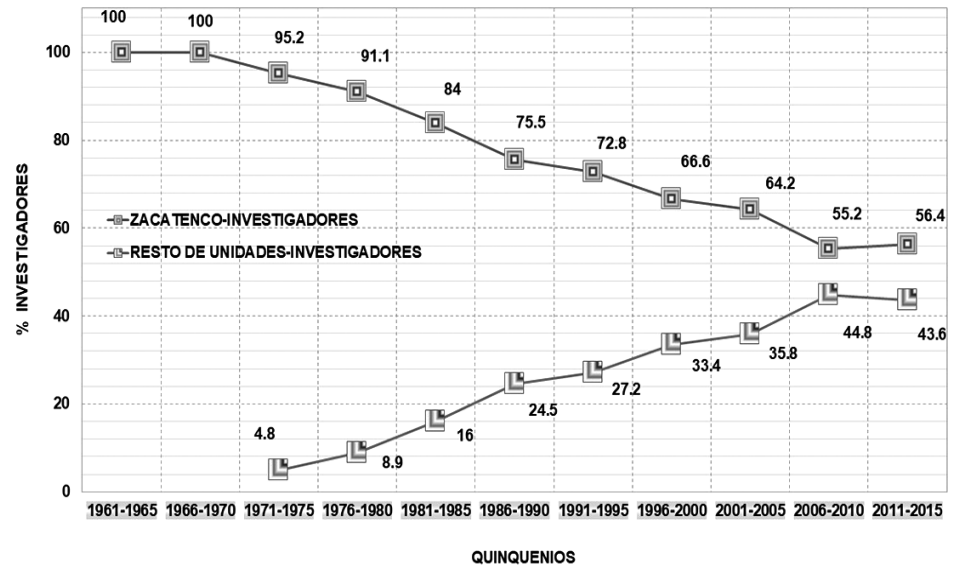

Figura 5. Porcentaje de investigadores en Zacatenco vs. los integrados en el resto de las unidades: 1961-2015

\section{Investigadores por género}

Las mujeres representan $25 \%$ del total de los profesores contratados por el Cinvestav. Por unidades y de acuerdo con la Figura 6, en Zacatenco se encuentra el mayor número de investigadoras $(54 \%)$ del total de las mujeres. La presencia de académicas en el resto de las unidades es escasa, excepto en Sede Sur, Irapuato y Mérida donde se contabilizaron 26, 17 y 16, respectivamente. Las demás unidades incluyen menos de siete y en otras ninguna, como es el caso de Tamaulipas. Lo anterior seguramente se debe a que el 
Cinvestav desarrolla disciplinas donde las mujeres tienen escasa presencia, como las ciencias exactas e ingenierías. Este fenómeno no es propio de una institución en particular, se trata de un suceso que se representa de la misma manera en distintos ámbitos, nacional, regional e internacional (Luna-Morales y Luna-Morales, 2018; Unesco, 2017). Diversos estudios demuestran que, a pesar de los esfuerzos que están realizando las mujeres por integrarse en estos campos de estudio, y aunque han comenzado a registrar mayor presencia, sigue siendo insuficiente (Luna-Morales y Luna-Morales, 2018).

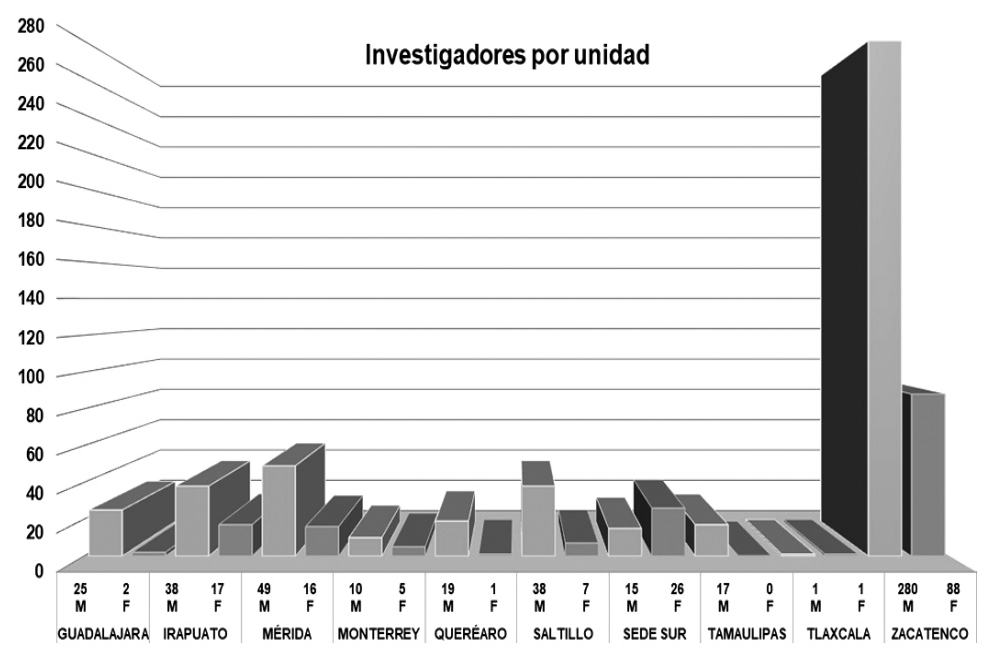

Figura 6. Distribución de investigadores del Cinvestav por unidades y género: 1961-2015

\section{Departamentos, Laboratorios y Programas}

De acuerdo con la Figura 7, Zacatenco es la sede con mayor número de departamentos. Sin embargo, en conjunto todas las unidades externas están contribuyendo de manera extraordinaria al ampliar el número de departamentos, secciones y laboratorios; en particular, Querétaro, Guadalajara, Saltillo y Monterrey, que se iniciaron con un solo laboratorio, actualmente se componen de cinco y cada uno integra su propio grupo de investigadores y temas de investigación. 


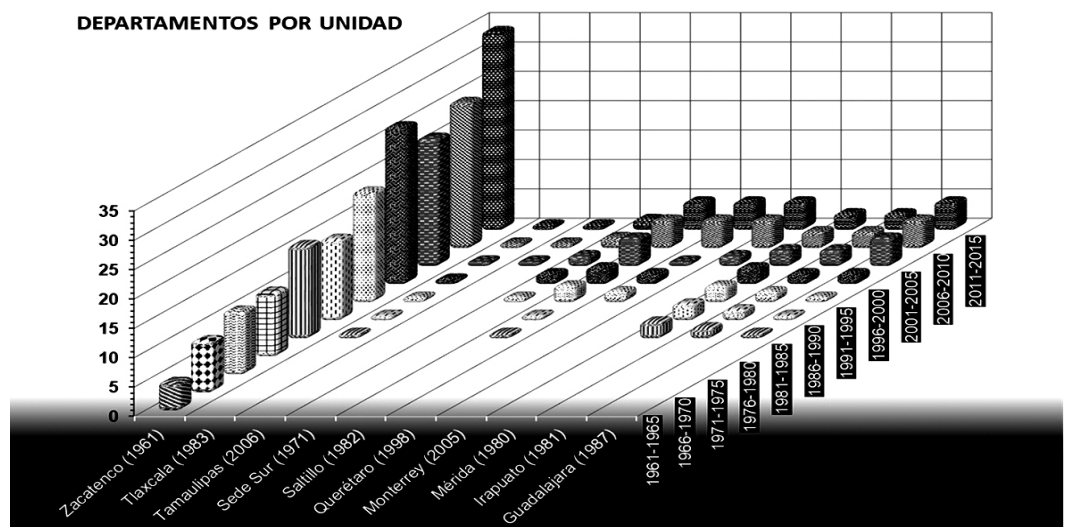

Figura 7. Estructura departamental por unidades y quinquenios: 1961-2015

\section{Graduados: maestría y doctorado}

Las Figuras 8 y 9 presentan a los graduados de maestría y doctorado en Zacatenco en comparación con las unidades. La Figura 8 incluye los graduados de maestría, donde Zacatenco contribuye de manera aislada hasta la primera mitad de la década de los años 70. En el quinquenio 1976-1980 se modifica porque aparecen las primeras contribuciones del Departamento de Investigaciones Educativas que se crea en 1971 y en 1975 incorpora sus programas de posgrados de maestría (Gutiérrez-Serrano, 1999). A estos esfuerzos se suman los de la Sección de Matemática Educativa fundada en 1975 (Centro de Investigación y Estudios Avanzados del IPN, 1971). En este sentido, $4.3 \%$ de graduados corresponden a la especialidad en matemática educativa.

En los siguientes quinquenios las unidades en provincia, incluida Sede Sur, empiezan a graduar de manera impresionante, al grado que en la etapa 2001-2005 están muy cerca de igualar a Zacatenco. Esto quiere decir que las unidades externas están contribuyendo con un mayor número de graduados. No se encuentra una explicación para justificar la separación de los últimos dos quinquenios, posiblemente tiene que ver con la matrícula de nuevo ingreso y egreso, donde el Cinvestav muestra una baja de titulados de maestría desde finales de los años 90 (Cinvestav, 2010).

La Figura 9 muestra los graduados de doctorado, se observa que las unidades externas registran sus primeras aportaciones en la segunda mitad del periodo de los años 80 . Mérida e Irapuato contribuyen con $3.2 \%$ en el quinquenio 1986-1990. Con la incorporación de nuevas unidades y el fortalecimiento 
de las existentes, en los quinquenios posteriores se incrementan los grados de doctorado; este esfuerzo se ve reflejado en los porcentajes alcanzados, en particular $46.7 \%$ en 2006-2010. Esto demuestra que las unidades lograron desarrollar programas de posgrado de mayor interés para los jóvenes en las diversas entidades federativas donde se ubican, todos reconocidos como PNPC validados por el Conacyt como de los mejores en el ámbito nacional. De esta manera contribuyen en conjunto con la unidad Zacatenco en el cumplimiento de los objetivos de la institución, al formar más de 40 \% de los graduados registrados en los últimos quinquenios del estudio.

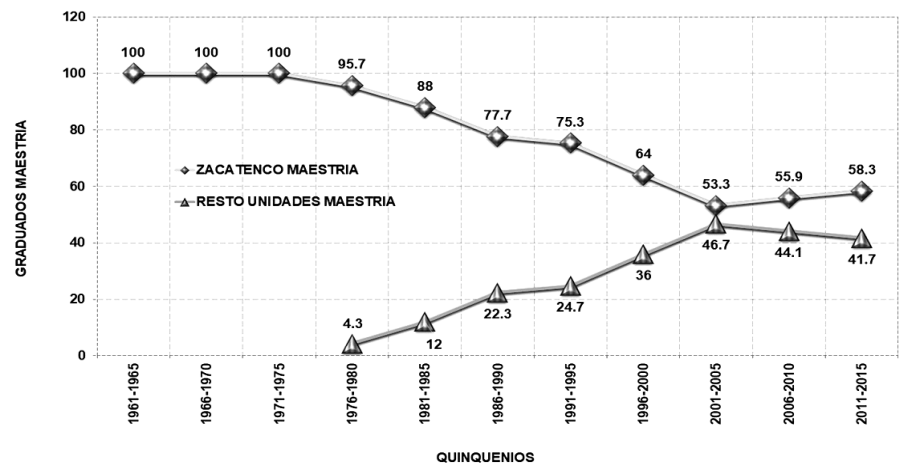

Figura 8. Graduados de maestría Zacatenco vs. resto de las unidades: 1961-2015

\section{Distinciones y reconocimientos}

La Figura 10 indica que es a partir de los años 1976-1980 cuando se presentan los primeros reconocimientos para las unidades externas; en el transcurso de los años se incrementan los porcentajes y concluye el periodo de estudio con 27.9 \%. Según Bourdieu (2003), los investigadores tienen un momento para concretar su capital científico, lo que se traduce en consolidación y reconocimiento. En este sentido, Zacatenco cuenta con los profesores de mayor antigüedad, quienes concretaron los objetivos de la institución, los de mayor liderazgo, los más consolidados en la producción e impacto científico, más líneas de investigación, departamentos, laboratorios y programas. Sin embargo, esto no quiere decir que las unidades externas no cuenten con estos perfiles, los hay sobre todo en las que tienen una trayectoria más larga. Por otro lado, no hay que perder de vista que muchos de los investigadores instalados en provincia se formaron en el Cinvestav bajo la dirección de profesores que tienen amplio reconocimiento y prestigio. 


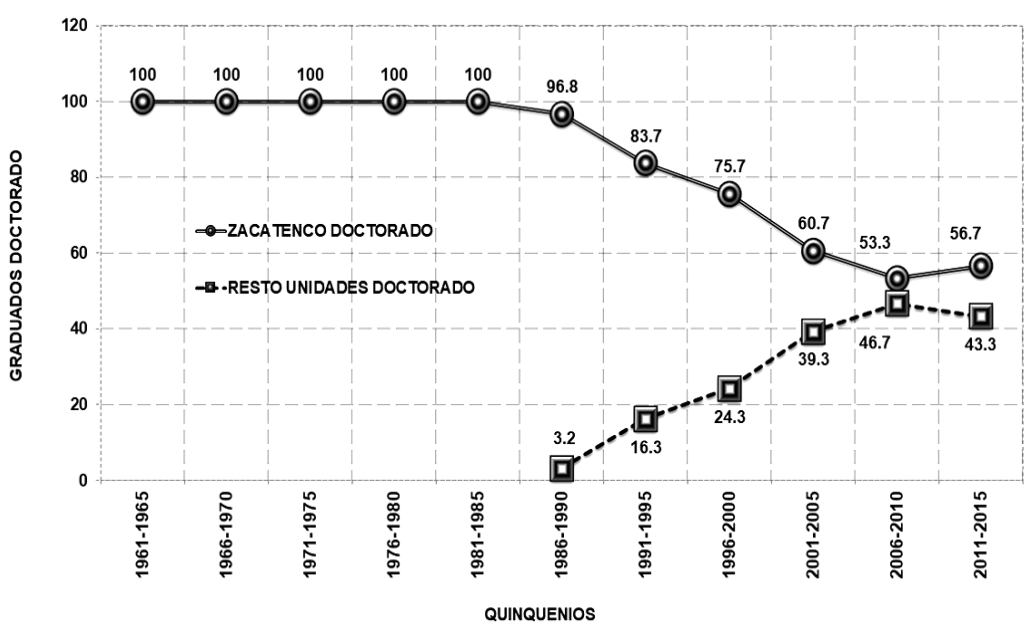

Figura 9. Graduados de doctorado Zacatenco vs. resto de las unidades: 1961-2015

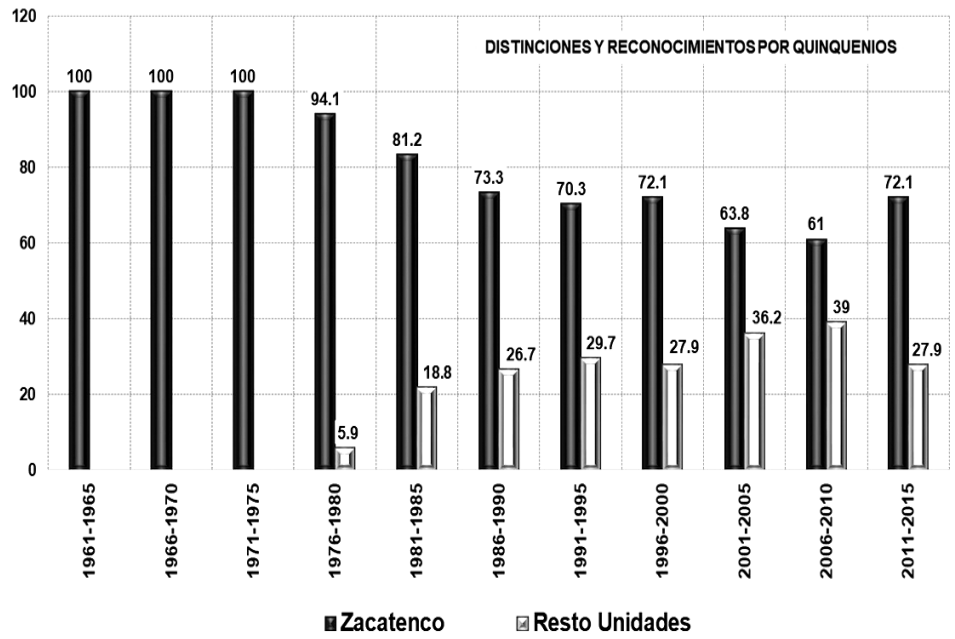

Figura 10. Distinciones y reconocimientos Zacatenco vs. resto de las unidades: 1961-2015 


\section{Presencia en años, producción y citas por dependencia del Cinvestav}

La Tabla 1 muestra, por unidad, los departamentos, laboratorios y programas que cada una integra, los años que tienen realizando investigación según los trabajos registrados en WoS y el área en la que se ubican, así como los promedios de trabajos y citas por año y por trabajo.

En Zacatenco se encuentran los departamentos con mayor presencia en años (entre 44 y 54), entre los que sobresalen los más productivos y citados, integrados a las líneas de investigación de mayor tradición en el centro: física, química, ingenierías, fisiología, biofísica y neurociencias; distribuidos en áreas de CBS, CEN y TCI. De acuerdo con WoS, el Cinvestav está constituido por 76 dependencias que cubren las cuatro áreas de estudio, donde las tecnológicas abarcan $50 \%$ del global.

Como se observa, las unidades externas que sobresalen es porque tienen mayor tiempo en la investigación, Tlaxcala, Mérida e Irapuato con más de 30 años y Sede Sur y Saltillo con más de 25, son las primeras en crearse y las que más alto promedio de trabajos y citas consiguen. Cabe mencionar que sus departamentos, al igual que los de otras unidades, integran campos de estudio que se extendieron de Zacatenco hacia las entidades federativas como es el caso de biotecnología, física, matemáticas e ingenierías, aunque también hay departamentos que se insertaron por interés de las autoridades de la institución o como resultado de la colaboración que mantienen con otras instituciones y países. No obstante, gran parte de los departamentos y laboratorios se fundaron para coadyuvar en el desarrollo de la industria instalada en la región y en la apertura de líneas de investigación que en México requieren de atención y explotación por los beneficios que ofrecen a la sociedad: recursos del mar, ecología humana, ingeniería genética, control automático, computación, ingeniería de sistemas, telecomunicaciones, diseño electrónico, investigación e innovación tecnológica, entre otras.

Cabe aclarar que $38 \%$ de los departamentos y laboratorios tienen menos de 10 años de haberse conformado. La mayor parte no presentan aportaciones significativas, lo que sugiere que van a tardar, sobre todo, en consolidar las estructuras de organización y contar con los líderes que logren reconocimiento internacional a través de las publicaciones científicas y la formación de nuevos recursos en la investigación. 


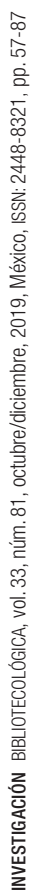

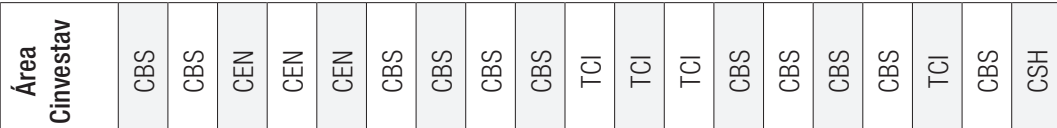

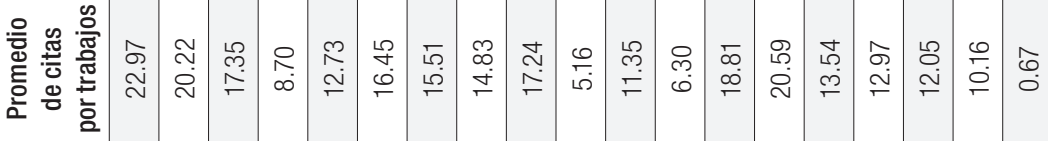

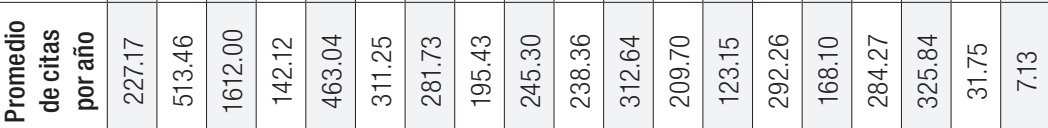

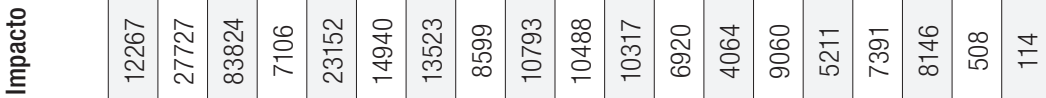

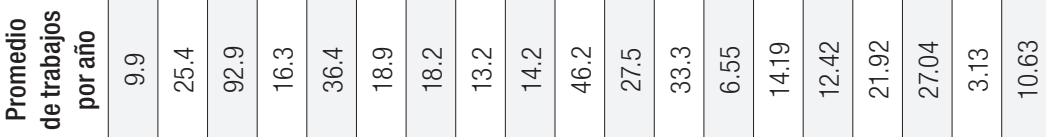

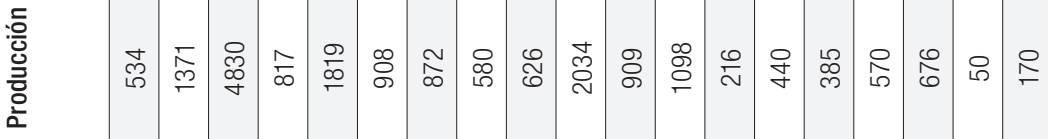
끙 흉 :드

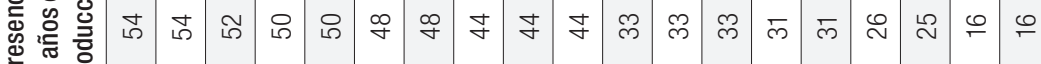

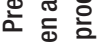

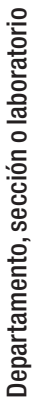

\begin{tabular}{|c|c|c|c|c|c|c|c|c|c|c|c|c|c|c|c|c|c|c|c|}
\hline 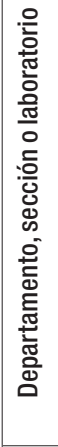 & 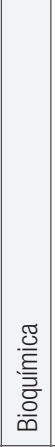 & 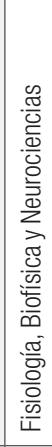 & 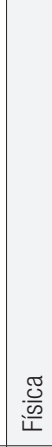 & 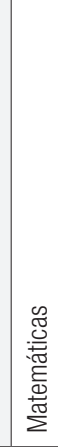 & 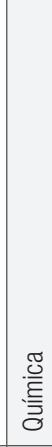 & 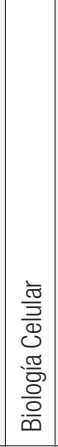 & 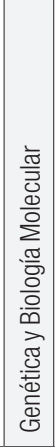 & 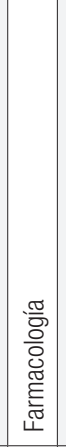 & 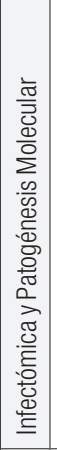 & 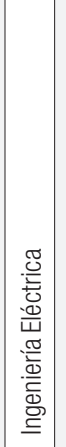 & 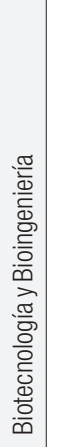 & 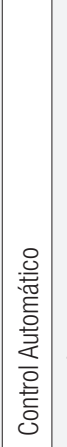 & 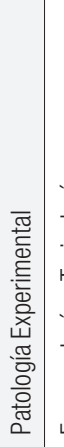 & 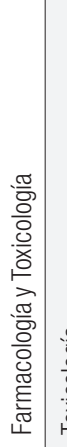 & 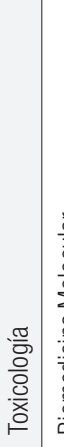 & 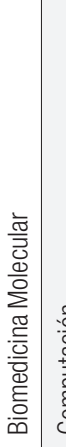 & 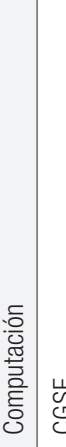 & $\begin{array}{c}\mathrm{u} \\
\mathrm{c} \\
\mathrm{c}\end{array}$ & \\
\hline 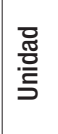 & 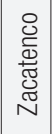 & 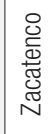 & 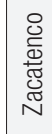 & 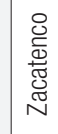 & 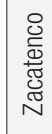 & 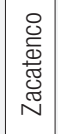 & 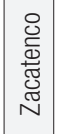 & 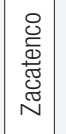 & 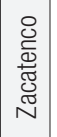 & 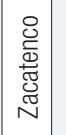 & $\begin{array}{l}\stackrel{0}{0} \\
\stackrel{\bar{d}}{0} \\
\overline{\mathbb{J}} \\
\mathbb{N}\end{array}$ & 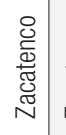 & 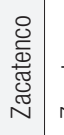 & 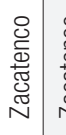 & 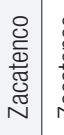 & 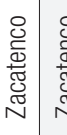 & 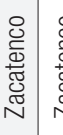 & 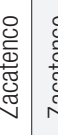 & \\
\hline 㕇 & - & $\sim$ & $m$ & $\nabla$ & ما & 0 & $\sim$ & $\infty$ & $\sigma$ & 으 & $\mp$ & $\cong$ & 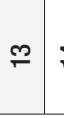 & 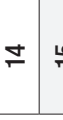 & 10 & $\stackrel{\circ}{\circ}$ & $\approx$ : & क & \\
\hline
\end{tabular}




\begin{tabular}{|c|c|c|c|c|c|c|c|c|c|c|c|c|c|c|c|c|c|c|c|}
\hline $\begin{array}{l}\mathscr{D} \\
\mathcal{O}\end{array}$ & D & I & 工 & D & $\bar{\wp}$ & 甹 & $\begin{array}{l}\mathscr{D} \\
\mathscr{D} \\
0\end{array}$ & 工्工 & 志 & $\bar{\varrho}$ & $\begin{array}{l}\mathscr{0} \\
\text { O) }\end{array}$ & 工्ञ & 工्ञ & $\bar{\varrho}$ & $\begin{array}{l}\mathscr{D} \\
\text { Ő }\end{array}$ & $\begin{array}{l}\mathscr{D} \\
\mathbb{0}\end{array}$ & $\bar{\vdash}$ & $\bar{\odot}$ & $\bar{\vdash}$ \\
\hline $\begin{array}{l}\underset{ \pm}{ \pm} \\
\stackrel{\sim}{ \pm}\end{array}$ & 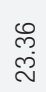 & $\begin{array}{l}\infty \\
\infty \\
\sim\end{array}$ & 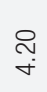 & $\frac{\hat{\sigma}}{\sigma}$ & $\begin{array}{l}\stackrel{L}{\rho} \\
0 \\
0\end{array}$ & $\stackrel{\infty}{\stackrel{\infty}{\sim}}$ & $\begin{array}{l}m \\
\stackrel{m}{\sigma} \\
\dot{q}\end{array}$ & $\begin{array}{l}8 \\
\circ \\
0\end{array}$ & $\begin{array}{l}\hat{\theta} \\
\dot{m}\end{array}$ & 0 & $\stackrel{\hat{\theta}}{\tilde{\theta}}$ & $\begin{array}{l}\text { లె } \\
\stackrel{\sim}{\sim}\end{array}$ & 0 & 0 & $m$ & $\begin{array}{l}\widetilde{D} \\
\infty \\
\underset{ \pm}{+}\end{array}$ & $\stackrel{\mathscr{C}}{\underset{C}{\leftarrow}}$ & $\stackrel{\stackrel{\sim}{\sim}}{+}$ & $\begin{array}{l}\infty \\
\infty \\
\dot{m}\end{array}$ \\
\hline$\frac{\infty}{\infty}$ & 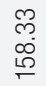 & $F_{\circ \circ}^{\circ}$ & $\begin{array}{l}\stackrel{2}{N} \\
\llcorner \\
\llcorner\end{array}$ & 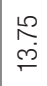 & $m$ & $\frac{N}{i \circ}$ & $\begin{array}{l}0 \\
10 \\
\\
i n\end{array}$ & 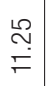 & $\begin{array}{l}\hat{U} \\
\dot{\sim} \\
\end{array}$ & 0 & $\begin{array}{l}\text { mె } \\
\underset{\sim}{\varpi}\end{array}$ & $\begin{array}{l}\tilde{m} \\
\text { } \\
\stackrel{\sim}{*}\end{array}$ & 0 & 0 & m & 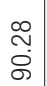 & 암 & $\widehat{\hat{\omega}}$ & 웜. \\
\hline হ্ণ & $\stackrel{\stackrel{N}{\mathbb{V}}}{\underset{\sim}{2}}$ & $\mathscr{q}$ & $\Im$ & $\stackrel{ }{=}$ & $\stackrel{\infty}{\ulcorner}$ & க & $\underset{\sim}{\bar{m}}$ & $\stackrel{\llcorner}{q}$ & 寸 & 0 & के & $\overline{6}$ & 0 & 0 & $m$ & $\begin{array}{l}\mathscr{D} \\
\infty \\
\infty\end{array}$ & $\underset{m}{\vec{m}}$ & ले & 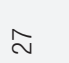 \\
\hline $\begin{array}{l}\mathscr{L} \\
\stackrel{\circ}{0} \\
0\end{array}$ & $\begin{array}{l}\infty \\
\substack{0 \\
0}\end{array}$ & $\stackrel{\infty}{\stackrel{\infty}{\leftarrow}}$ & $\stackrel{\stackrel{2}{\sim}}{\leftarrow}$ & $\stackrel{\circ}{\circ}$ & $\begin{array}{l}\llcorner 0 \\
\infty \\
\infty\end{array}$ & $\sim$ & $\stackrel{m}{\stackrel{m}{-}}$ & 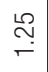 & $\nabla$ & $\sim$ & $\sim$ & - & $\sim$ & $\sim$ & - & $\begin{array}{l}8 \\
0 \\
0\end{array}$ & $\begin{array}{l}\stackrel{\rho}{\infty} \\
\stackrel{0}{\Gamma}\end{array}$ & $\stackrel{m}{\stackrel{m}{-}}$ & 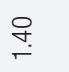 \\
\hline is & $\overline{6}$ & $\stackrel{\circ}{\leftarrow}$ & 으 & $\simeq$ & ¿م & $\simeq$ & $\infty$ & 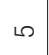 & $\simeq$ & 0 & 0 & m & $\sim$ & $\sim$ & - & $\stackrel{\llcorner}{\circ}$ & 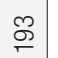 & $\infty$ & $\curvearrowright$ \\
\hline$\sigma$ & $\infty$ & $\sigma$ & $\infty$ & $\infty$ & 0 & 0 & 0 & $\nabla$ & $m$ & $m$ & ๓ & ल & - & - & - & $\widetilde{m}$ & 으 & 0 & $L 0$ \\
\hline 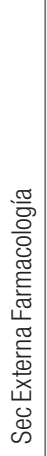 & 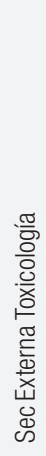 & 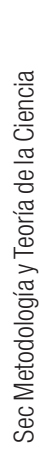 & $\begin{array}{l}\infty \\
\text { J్ } \\
\text { U. }\end{array}$ & 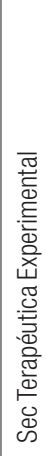 & 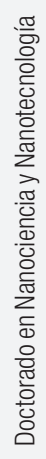 & 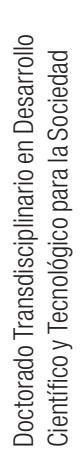 & 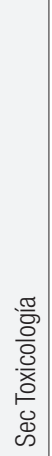 & 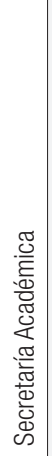 & 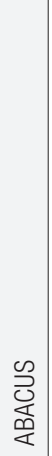 & $\begin{array}{l}0 \\
\bar{E} \\
\text { J }\end{array}$ & 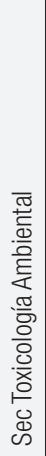 & 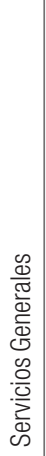 & 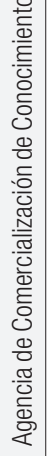 & 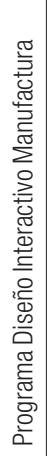 & 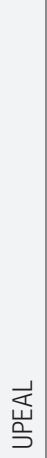 & $\underset{\frac{\pi}{0}}{\frac{\mathbb{c}}{0}}$ & 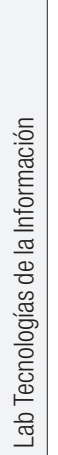 & 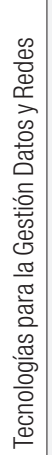 & 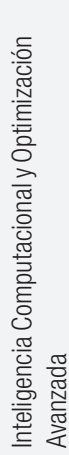 \\
\hline 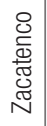 & 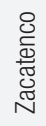 & $\begin{array}{l}\stackrel{O}{0} \\
\overline{\tilde{J}} \\
\text { त्य } \\
\mathbb{J} \\
N\end{array}$ & 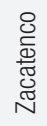 & 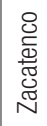 & 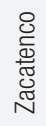 & 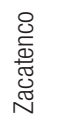 & 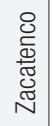 & 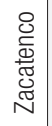 & 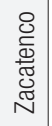 & 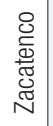 & 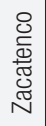 & 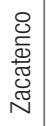 & 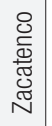 & 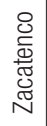 & 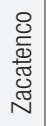 & 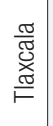 & 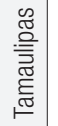 & 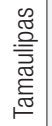 & 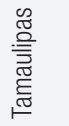 \\
\hline ని & $\bar{\sim}$ & ล & ๗ & d & బ & $\stackrel{N}{2}$ & $\approx$ & $\stackrel{\infty}{\sim}$ & న & ஜి & க & న్ & ణ్ల & ষ & 뉴 & $\ddot{m}$ & 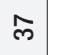 & $\stackrel{\infty}{\infty}$ & శ్ల \\
\hline
\end{tabular}




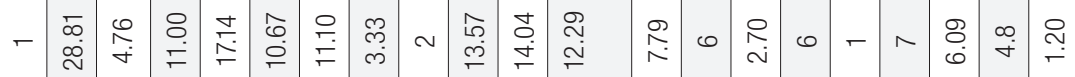

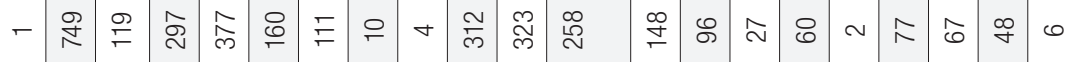

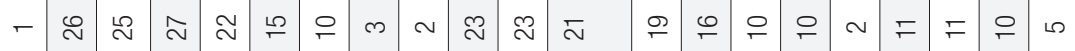




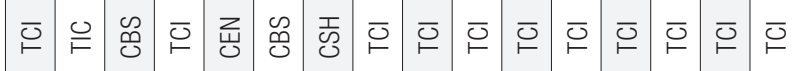

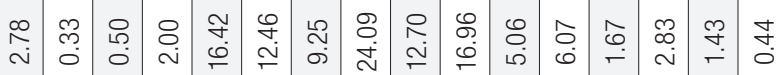

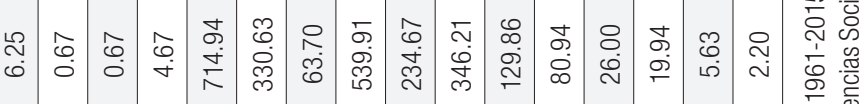

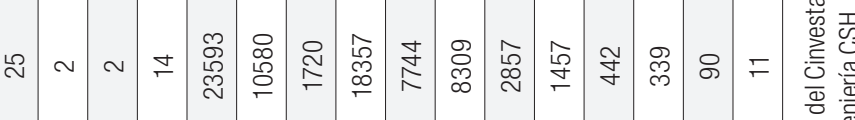

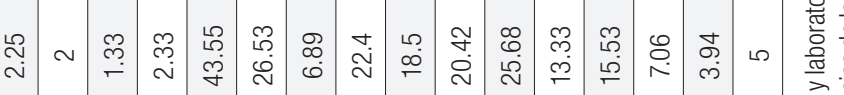

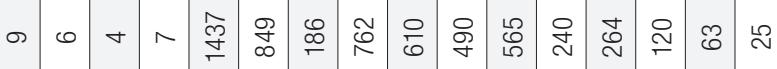
क्षे 


\section{Trabajos más citados, investigadores, departamentos y reconocimientos por unidad}

La Figura 11 presenta la distribución de trabajos con 100 o más citas por unidades, así como el número de investigadores, departamentos, laboratorios y programas, posgrados reconocidos por el Conacyt, premios y reconocimientos. Cabe mencionar que Monterrey, Saltillo y Tamaulipas no forman parte de la figura porque no reúnen este tipo trabajos.

Como se puede observar, Zacatenco sobresale en todas las variables analizadas, pese a ello, es importante señalar el rol que juegan las unidades Mérida, Irapuato y Sede Sur que están contribuyendo de manera favorable al crecimiento del Cinvestav mediante el número de trabajos, citas, investigadores, posgrados, premios y reconocimientos. Querétaro y Guadalajara se imponen por los departamentos que incorporan. Tlaxcala cuenta con una larga trayectoria en la reproducción de animales, no registra posgrados y generalmente se ha mantenido con uno o dos investigadores, entre ellos, Carlos Beyer Flores quien con su prestigio logró conseguir trabajos altamente citados. Un punto a considerar es la trayectoria que presentan Guadalajara y Querétaro, cuyas aportaciones son de las más bajas entre los indicadores analizados.

Saltillo no muestra trabajos con 100 o más citas, aun cuando es la cuarta unidad que incorpora más investigadores. En este sentido, se esperaría que formara parte de la figura; sin embargo, por los temas de investigación que cubre, el tipo de productos que generan no son de los más citados. Es el mismo caso de la unidad Guadalajara, Tamaulipas, Sede Sur y parte de las líneas de investigación de Querétaro y Zacatenco.

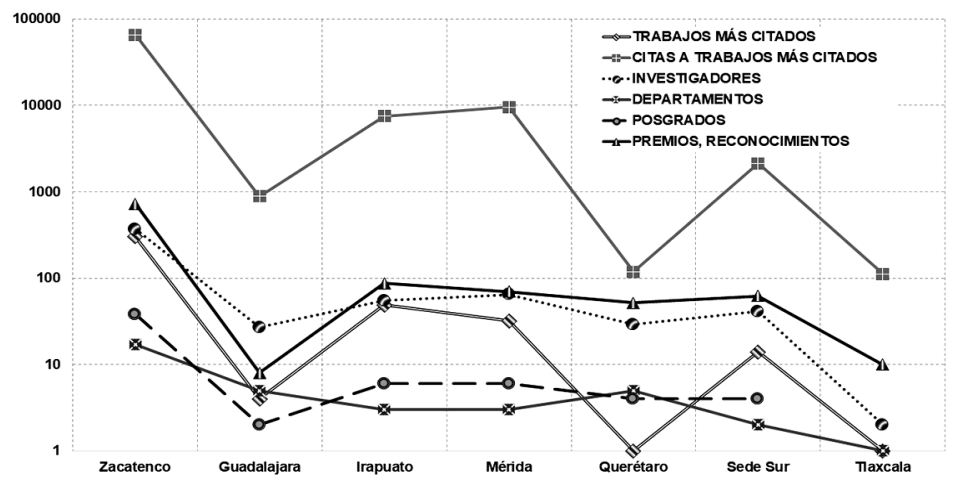

Figura 11. Distribución de trabajos más citados, investigadores, departamentos, posgrados, premios y reconocimientos por unidades: 1961-2015 


\section{Discusión}

Las medidas de descentralización implementadas durante 1970-2000 generaron impactos importantes para el país y para las instituciones participantes (Larqué-Saveedra, 2016), sobre todo las últimas, que aprovecharon para expandirse con nuevas sedes en distintos estados del país.

Este estudio demuestra que el Cinvestav se está descentralizando desde hace 40 años y que las unidades están contribuyendo de forma muy sustancial al desarrollo del país. Esto no quiere decir que en Zacatenco se esté trabajando menos, considerando que en esta unidad se encuentran los investigadores que surgieron con la institución, los que le dieron vida y la convirtieron en uno de los nichos científicos más reconocidos en los diversos ámbitos, sobre todo por la formación de nuevos recursos y la generación de investigación en distintos campos de estudio. Es por lo anterior que se mantienen los promedios de trabajos y citas más altos, trabajos más citados, posgrados de calidad, premios y reconocimientos.

Parte de los avances que ha logrado el Cinvestav tienen que ver con la consolidación de sus diferentes campos de investigación: biotecnología y bioquímica, recursos del mar, cerámica y metalúrgica, computación y tecnologías, biología, química, matemáticas y física, entre otras. Esta última, identificada como la más productiva, de acuerdo con Collazo-Reyes y Luna-Morales (2002), exhibe aspectos claros de un proceso de maduración exitoso.

Por otro lado, se debe mencionar que hay temas de investigación que están logrando impactar entre la comunidad científica nacional e internacional, como el estudio de la secuencia de los genomas complejos, desarrollado en el Laboratorio Nacional de Genómica para la Biodiversidad (Langebio). Este grupo se ha integrado a proyectos como The 1000 Genomes Project Consortium que se propuso ofrecer una descripción completa de la genética humana (Genome Project Consortium, 2015). Una participación similar muestran las ingenierías con el cómputo evolutivo, que también ha logrado interés entre los pares; al igual que la física de partículas y campos que desde mitad de los años 90 están participando en el descubrimiento de nuevas partículas: top quark y partícula de Higgs. No obstante, estas no son las únicas razones por las que se esperan crecimientos en las unidades del Cinvestav. Sin duda, lo más importante es la consolidación de los grupos de investigación, así como la conformación de las estructuras organizacionales acordes a las necesidades de cada unidad, departamentos, laboratorios y secciones (Bourdieu, 2003); lo que finalmente se ve traducido en conocimiento y reconocimiento, reflejado a través de la formación de recursos, producción científica y citas registradas en los índices de citas internacionales. 
González-Quiroz y Remedi-Allione (2013) dicen que el liderazgo y fortalecimiento de un investigador en el Departamento de Biología Celular del Cinvestav se alcanza en 10 años. Quizá valga la pena ver cuál es el tiempo de los otros campos de investigación pues podría ayudar a entender los procesos de maduración de la comunidad académica, así como el estudio de áreas o líneas de investigación específicas que por años se han mantenido de manera exitosa en el Cinvestav.

Las mujeres investigadoras y su participación en el desarrollo de la ciencia es un tema en discusión desde hace años, sobre todo, por la escasa presencia que tienen en campos como las ciencias exactas e ingeniería (Valle Díaz Muñoz y Garay-Sánchez, 2012). El Cinvestav cubre su planta académica y de investigación con una cuarta parte de investigadoras, la mayoría en las áreas de ciencias biológicas y sociales. Pese a las limitaciones en estudios de género, el Cinvestav destaca como una de las instituciones que mayor número de investigadoras integra, incluso en los campos considerados masculinos (Luna-Morales y Luna-Morales, 2018).

Uno de los problemas más graves por los que atraviesan gran parte de las instituciones es la falta de recursos, un determinante que afecta en distintos aspectos, incluso plazas para investigadores. De acuerdo con los resultados, el Cinvestav desde hace 10 años prácticamente no contrata investigadores, consecuencia de las políticas públicas aplicadas en el país que no garantizan recursos suficientes para el desarrollo de la ciencia y la tecnología (CEPAL, 2016).

El Cinvestav es de las instituciones nacionales que integran mayor número de PNPC (maestría y doctorado), distribuidos entre sus distintas unidades. Esto explica el incremento en el número de graduados, sobre todo, si son de interés en contenido y se ajustan a los requerimientos de la región donde se imparten. Complementa lo anterior la presencia de investigadores líderes en temas específicos de investigación, es decir, aquellos que han conseguido premios y reconocimientos nacionales e internacionales contribuyendo al prestigio de la institución.

\section{Conclusiones}

De acuerdo con lo registrado en WoS, el Cinvestav mantiene crecimientos resultado del esfuerzo que realizan las diversas unidades que lo conforman, de donde se infiere que este centro ha consolidado sus estructuras de organización tomando en cuenta, entre otros factores, el cumplimento de los objetivos, la capacidad de organización y coordinación del trabajo, jerarquías, toma de decisiones, solución a problemas, productividad y competitividad 
(Rubio-Castillo, 2009). Es decir, es un sistema que integra normas establecidas y asumidas por la propia comunidad, definidas según la situación económica, política, social y cultural en la que se desenvuelve y cuenta con los líderes suficientes que coadyuven en la formación de recursos y actividad científica, dando lugar a uno de los nichos de investigación de mayor reconocimiento y prestigio internacional (Chavoya-Peña, 2002).

La descentralización ha dado resultados favorables para el Cinvestav. De acuerdo con Ibarrola (2002) y Luna-Morales (2013), mediante este proceso se han incorporado nuevas unidades, departamentos, secciones, laboratorios, programas de estudio e investigadores. De igual manera ha crecido el número de estudiantes graduados, la producción e impacto científico. El Cinvestav desde sus primeros 10 años se conformó como la institución científica más productiva en México y América Latina (Pérez-Tamayo, 2004).

El proceso de institucionalización y descentralización de la ciencia y la tecnología sin duda ha generado beneficios no sólo para el Cinvestav, sino también para otras instituciones del país (Larqué-Saveedra, 2016); sin embargo, las ventajas alcanzadas no son del todo producto de este nuevo contrato que establece el Estado y la sociedad, también es fruto de los esfuerzos que han hecho las propias instituciones y grupos de investigación en un país donde el Estado no suele cumplir con la parte que le corresponde, es decir, otorgar recursos para lograr metas y beneficios (Azfar et al., 1999).

Este trabajo está orientado a mostrar indicadores cuantitativos simples; no obstante, hay otros enfoques que se pueden aplicar para determinar patrones de comportamiento en la actividad científica y tecnológica que se producen en la institución. En este sentido, consideramos conveniente abordar en un primer trabajo los aspectos generales y, en investigaciones posteriores, conducir los enfoques hacia los niveles micro, por unidad, para caracterizar con detalle la forma en que se desenvuelven y contribuyen en la región donde se localizan. De esta manera, precisar la forma en que participan a través de dos vertientes: 1) líneas de investigación que cubren, vinculación que tienen con la industria de la región y los benéficos que aportan a la sociedad; 2) identificar los patrones de producción de conocimiento que generan los distintos campos de estudio que cubre el Cinvestav. Este aspecto no se ve reflejado en el estudio actual, donde las ciencias sociales y las ingenierías tradicionalmente tienden a presentar resultados como patentes, reportes de investigación, técnicos y de asesoría, libros y capítulos de libros, entre otros productos, y en cuyos casos se requiere de la aplicación de bases de datos más específicas o la construcción de una propia que integre los indicadores apropiados para el análisis. De esta manera, se medirían de manera más completa las aportaciones de las unidades del Cinvestav, dando 
lugar a patrones de producción más completos. Resulta interesante además comparar al Cinvestav con otros centros similares a nivel nacional, regional e internacional, como una manera de identificar comportamientos en términos de producción, impacto, posgrados, graduados, redes de colaboración, entre otras variables de análisis. Sin duda se trata de un trabajo más amplio que implica conocer tanto al Cinvestav como a la institución con la que se va a comparar.

En términos generales, la hipótesis se cumple: Zacatenco logra mayores aportaciones y reúne el más alto número de investigadores, entre ellos a los primeros profesores contratados por el Cinvestav, comprometidos en convertir a esta institución en una de las más reconocidas en todos los ámbitos. Es la unidad que integra más departamentos, secciones, laboratorios y programas, y la que más distinciones y reconocimientos ha logrado.

\section{REFERENCIAS}

Azfar, O., S. Kahkonen, A. Lanyi, P. Meagher y D. Rutherford. 1999. "Decentralization, Governance and Public Services the Impact of Institutional Arrangements", en Devolution and Development. Govemance propspects in Decentralizing States, editado por S. Kimenyi y Patric Meacher. Maryland: IRIS Center, University of Maryland. https://www.taylorfrancis.com/books/e/9781351160001/chapters/10.4324/9781351160001-13

Bourdieu, P. 2003. El oficio del científico: ciencia de la ciencia y reflexividad, Barcelona: Anagrama.

Cabrero-Mendoza, E. 2000. "Los dilemas de la descentralización en México". Organizações \& Sociedade 7 (19): 123-152.

Campos-Ríos, G. y M. E. Martínez de Ita. 2011. "México. La ley de conservación del centralismo en la investigación. Principales efectos". Revista de la Educación Superior 15 (2): 137-150.

Centro de Investigación y de Estudios Avanzados del IPN. 1971. Anuario Cinvestav. México: Cinvestav.

CEPAL (Comisión Económica para América Latina y el Caribe). 2016. Ciencia, tecnología e innovación en la economía digital. La situación de América Latina, documento para la Segunda Reunión de la Conferencia de Ciencia, Innovación y TIC de la CEPAL, San José, septiembre 12-13. Santiago de Chile: CEPAL, Unesco, Naciones Unidas.

Chavoya-Peña, M. L. 2002. "La institucionalización de la investigación en ciencias sociales en la Universidad de Guadalajara". Revista de la Educación Superior 31 (121): 7-25.

Cinvestav (Centro de Investigación y de Estudios Avanzados). 2010. Atlas de la ciencia en el Cinvestav. 1961-2010. Fecha de consulta: 02 de enero de 2019. https:// www.fis.cinvestav.mx/atlas/flash.html

Collazo-Reyes, F. y M. E. Luna-Morales. 2002. "Física mexicana de partículas elementales: organización, producción ciéntifica y crecimiento". Interciencia 27 (7): 347-353. 
Fernández-Ruíz, J. 2010. La descentralización política y las universidades públicas de los estados. México: UNAM, Instituto de Investigaciones Jurídicas.

García-Ramos, J. 1970. Alocución del Doctor Juan García Ramos: Anuario Cinvestav 1970: Ceremonia de Homenaje en Memoria del Señor Doctor Arturo Rosenblueth. México: Cinvestav.

Genome Project Consortium. 2015. "A Global reference for human genetic variation”. Nature 526 (october): 68-87.

González-Quiroz, J. y E. Remedi-Allione. 2013. "La formación y consolidación de investigadores en el Cinvestav: una mirada desde uno de sus departamentos”, trabajo presentado en el XII Congreso Nacional de Investigación Educativa, Universidad de Guanajuato, México, 18-22.

Gutiérrez-Serrano, N. G. 1999. Redes, comunidades, grupos y trabajo entre pares en la investigación educativa. México: Cinvestav, Departamento de Investigaciones Educativas, Plaza y Valdés.

Gutiérrez-Serrano, N. G. 1997. "Orígenes de la institucionalización de la investigación en México”. Tesis de maestría, Cinvestav, Investigaciones Educativas.

Hernández-Arias, A. 2013. "Informe sobre el índice de impacto de las revistas científicas”. Compendium 16 (30): 95-115.

Hernández-García, Y. y H. Navarro-Contreras. 2017. "Estudio bibliométrico del sistema de investigación local de San Luis Potosí (1920-2015)”. Investigación Bibliotecológica 31: 249-270.

Huerga-Melcón, P. 2012. "Luces y sombras de la Revolución Científico Técnica. Notas críticas sobre la cuestión del declive de los estados". Nómadas. Crítica de Ciencias y Jurídicas 35: 1-14. Fecha de consulta: 13 junio de 2017. http://webs.ucm.es/ info/nomadas/35/pablohuerga_2.pdf

Ibarrola, M de. 2002. "La impronta genética del Cinvestav. Una mirada a la excelencia de excelencia de la institución entonces y ahora", en El Cinvestav: trayectoria de sus departamentos, secciones y unidades: 1961-2001, 11-54. México: Cinvestav.

Illán-Sailer, J. C. 2006. Los procesos de descentralización y los retos para la ayuda internacional. Madrid: FIIAPP, Universidad Complutense de Madrid.

Jaramillo-Cardona, M. C. 2010. "La descentralización: una mirada desde las políticas públicas y las relaciones intergubernamentales en Baja California”. Región y Sociedad 22 (49): 177-200.

Larqué-Saavedra, A. 2016. "La descentralización de la ciencia en México". La Crónica, 14 de octubre, 2016.

López-Olmedo, R., R. Marmolejo-Leyva, M. A. Pérez-Angón, L. L. Villa-Vázquez y E. Zayago-Lau. 2017. "The role of public policies in the decentralization process of Mexican science and the formation of new researchers in institutions outside the Mexico City area". Scientometrics 112: 1343-1366.

Luna-Morales, E. 2013. "Desarrollo estructural institucional visto a través de indicadores bibliométricos”. Tesis de maestría, UNAM, Facultad de Filosofía y Letras, Instituto de Investigaciones Bibliotecológicas.

Luna-Morales, M. E. y E. Luna-Morales. 2018. "Mujeres investigadoras en las primeras estructuras de organización en ciencias exactas e ingenierías en México de 1900-2000: Estudio bibliométrico”. Investigación Bibliotecológica 32 (77): 193-215.

Martínez-Palomo, A. 2011. "Los primeros 50 años del Cinvestav". La Crónica, 13 de julio, 2011. Fecha de consulta: 13 junio de 2017. http://www.cronica.com.mx/notas/2011/591700.html 
Mendoza, J. y J. B. Garza. 2009. "La medición en el proceso de investigación científica: evaluación de validez de contenido y confiabilidad". InnOvaciOnes 6 (1): 17-32.

Merton, R. K. 1988. La sociología de la ciencia: investigaciones teóricas y empíricas. Recopilación e introducción de Norman W. Storer. Madrid: Alianza Editorial.

Peña-Ocando, D. 2011. "Algunas razones para evaluar la investigación científica venezolana desde la bibliometría”. Revista Interamericana de Bibliotecología 34 (3): 271-281.

Pérez-Tamayo, R. 2004. Historia General de la Ciencia en México en el Siglo XX. México: Fondo de Cultura Económica.

Pritchard, A. 1969. "Statistical bibliography on bibliometrics". Journal of Documentation 25 (4): 348-349.

Retana-Guiascón, O. G. 2009. "La institucionalización de la investigación científica en México. Breve cronología”. Ciencias 94 (abril-junio): 46-51.

Romo-Murillo, D. 2006. "El impacto de la ciencia y la tecnología en el desarrollo de México", en La ciencia y la tecnología como ejes de la competitividad de México, Adriana Borjas Benavente y Mónica Bucio Escobedo (coord.), 127-194. México: Centro de Estudios Sociales y de Opinión Pública.

Rubio-Castillo, F. A. 2009. "La estructura organizacional en centros de investigación, desarrollo e innovación; una aproximación a la experiencia internacional”. Tesis de doctorado, Universidad Autónoma de Querétaro, Facultad de Contaduría y Administración.

Saldaña-González, J. J. y L. F. Azuela-Bernal. 1994. "De amateurs a profesionales. Las sociedades científicas mexicanas en el siglo XIX”. Quipu. Latinoamericana de Historia de la Ciencia y la Tecnología 11 (2): 135-172.

Saldaña-González, J. J. 1990. "La ciencia y el debatían mexicano”. Actas de la Sociedad Mexicana de la Ciencia y la Tecnología I: 37-52.

Tinajero-Villavicencio, G. 2005. "Una década de acreditación de programas de posgrado: 1991-2001”. Revista de Educación Superior 34 (1): 107-120.

Unesco. 2017. Cracking the code: Girls' and women's education in science, technology, engineering and mathematics (STEM). París: Unesco.

Ureña, 2016. "Revolucionar la Biotecnología: Octavio Paredes López”. Cienciamx Noticias, México: Conacyt, Agencia Informativa, mayo, 2016. Fecha de consulta: 29 de marzo de 2018. http://www.conacytprensa.mx/index.php/sociedad/personajes/7117-biotecnologia-ipn-unam-cinvestav

Valle Díaz Muñoz Del, G. y A. Garay-Sánchez. 2012 “La falta de inclusión de mujeres en las ciencias exactas e ingenierías". Ciencia (julio-septiembre):34-43.

Vanti, N. 2010. "Métodos cuantitativos de evaluación de la ciencia: bibliometría e informetría”. Investigación Bibliotecológica 14 (29): 9-23.

Velasco, B., J. M. Eiros-Bouza, J. M. Pinilla y J. A. San Román. 2012. "La utilización de los indicadores bibliométricos para evaluar la actividad investigadora". Aula Abierta 40 (2): $75-84$. 
Para citar este texto:

Luna-Morales, María Elena, Evelia Luna-Morales y Miguel Ángel PérezAngón. 2019. "Los impactos de la descentralización de la enseñanza y la investigación en el Cinvestav de 1961-2015: estudio bibliométrico”. Investigación Bibliotecológica: archivonomía, bibliotecología e información 33 (81): 57-87.

http://dx.doi.org/10.22201/iibi.24488321xe.2019.81.57939 